\title{
Diminutive left-handed plasmonic nanoantenna-lens system in optical realm: ultraviolet emission and flat lens application
}

\author{
Monika RAJPUT ${ }^{1 *}$, Bhawana DABAS ${ }^{1}$, Than Singh SAINI ${ }^{2}$, NeEru MEHTA $^{1}$ \\ ${ }^{1}$ Ambedkar Institute of Technology, Directorate of Training and Technical Education \\ (Government of Delhi) \\ ${ }^{2}$ Optical Functional Materials Laboratory, Toyota Technological Institute, Nagoya 468-8511, Japan \\ *Corresponding author: dr.rajputmonika@gmail.com
}

\begin{abstract}
In present paper, a tunable left-handed plasmonic nanoantenna is designed to attain ultraviolet emission through a second harmonic generation with a phase harmonic condition. For the devised structure, the dispersion properties and negative values of the magnetic permeability and the electric permittivity show that the designed structure is left-handed for the primary wave (red light) and right -handed for the second harmonic wave (ultraviolet light). The 3D finite-difference time-domain method is employed to reveal its nonpareil skills (i.e. immense left-handed transmission efficiency and far-field spectrum exhibiting directionality). Attained results by numerical calculation for the second harmonic generation are accomplished with finite-difference time-domain analysis. The impact of physical parameters on transmission and dispersion characteristics is also scrutinized. Furthermore, flat lens application for a red light region with a centered wavelength at $\lambda=650 \mathrm{~nm}$ from the guileless design of LHM with no aberration is triumphed. Ultimately, a multifunction left-handed material is designed revealing tremendous potential to amass and abridge future applications in one architecture.
\end{abstract}

Keywords: left-handed materials, negative refraction, plasmonic optical nanoantenna, second harmonic generation, flat lens.

\section{Introduction}

In latest foregone time, negative refraction (NR) and negative index material (NIM) or left-handed material (LHM) in a low wavelength region gained mammoth attention in having a vision of their application in the design and development of photonic devices at nanoscale [1-17]. However, in the infra-red region nanoscale, split ring resonator (SRR) structures showed astonishing results. Even so, SRR endured with the saturation limit of a resonant magnetic response with negative effective permeability at high frequencies [14]. Subsequently, substitute strategies to design and develop contemporary materials baring NR have been of great interest of researchers [ $\underline{1}-\underline{14}]$. Ad- 
ditionally, striking advancement has been recently made toward shrinking the size of optical devices. This vogue toward compactness is propelled by the increase in system functionality and reduction in power dissipation. One more major inducement is a foresight of a design in which photonic circuits integrate flawlessly into bulky electronic systems. This necessitates waveguides that overpass the cavity between traditional micron-scale integrated photonics and nanoscale electronics. Furthermore, novel active devices can be developed utilizing robust nonlinear optical effects of nanostructured materials. Accordingly, numerous issues of wave propagation including nonlinear effects and surface modes in negative index materials (NIMs) are discussed [18-32]. Thus, in this paper, new frontiers are explored to demolish the inherent limitations of scaling for LHM designs to ultraviolet and optical wavelength region exhibiting nonlinear or tunable properties with nanophotonic device application, e.g. nanoantenna and imaging. However, to achieve tunability in LHMs, its nonlinear properties such as second harmonic generation (SHG) should be explored. The SHG in bulk LHM is discussed by LAPINE et al. [18]. The issue of SHG in LHM is further analyzed by researchers and attracted much more attention [ $\underline{18}-\underline{25}, \underline{32}]$.

In the current work, second harmonic generated ultraviolet light emanation is triumphed in afresh designed left-handed plasmonic nanoantenna (LHPNA). The biggest advantage of this work is that it presents the simplest design structure to achieve ultraviolet emission through SHG, which not only satisfies all the conditions for SHG proposed earlier but also keenly strengthened by the finite-difference time-domain (FDTD) analysis. Another feature of the structure is that we can tune the SHG for different wavelength of whole visible spectrum by judiciously engineering the structural parameters. Furthermore, designed LHM structure offers several functionalities, e.g. imaging, plasmonic optical nanoantenna, optical mirror and light ruling, etc.

Lorentz model is employed to characterize the semiconductor host (gallium arsenide) and Drude model for embedded metal (silver).

The harmonic generation is anticipated when for any wave, either primary or harmonic, structure becomes left-handed $[\underline{10}, \underline{19}, \underline{23}, \underline{24}]$. When a nonlinear primary wave

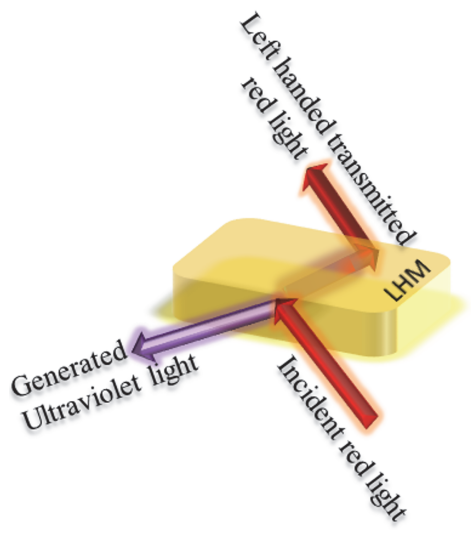

Fig. 1. Schematic of blue light generation and negative refraction for red light designed in LHMs. 
propagates in the structure, its nonlinear polarizability generates a wave with dual multiple frequency in a conflicting track as shown in Fig. 1.

Evaluation of dispersion properties and negative values of the magnetic permeability and the electric permittivity revealed that the designed structure is left-handed for the primary wave (PW) (red light) and right-handed for the second harmonic wave (SHW) (ultraviolet light). Contrary refractive indices for both waves give reverse energy propagation for produced harmonic. Negative real values of both permeability and permittivity with low imaginary values are obtained for the primary wavelength centered at $650 \mathrm{~nm}$. The field intensity pattern is also evaluated for PW and SHW for the structure using coupled Maxwell equations. The FDTD method is exerted to evaluate its transmission properties, where SHG is again validated, as depicted by the dispersion properties and mathematical analysis. Further FDTD simulation is performed and shows the application potential of the designed structure as flat lens. The 3D FDTD method is used to compute the near-field and far-field spectrums to predict important nanoantenna characteristics, i.e. directionality and efficiency.

\section{LHPNA: devise and scrutiny}

LHPNA structure is designed by a pair of silver nanorods embedded in a semiconductor $(\mathrm{GaAs})$ host material with a dielectric constant $\varepsilon_{\mathrm{h}}=13.25$.

The schematic of the LHM structure is shown in Fig. 2a, where $r$ having value of $0.31 \mu \mathrm{m}$, is the radius, and $a$ having the value $0.15 \mu \mathrm{m}$, is the separation between the nanorods. Permittivity values of host and embedded metal have been taken in account to evaluate the dispersion properties of the proposed structure and is shown in Fig. $2 \mathbf{b}$.
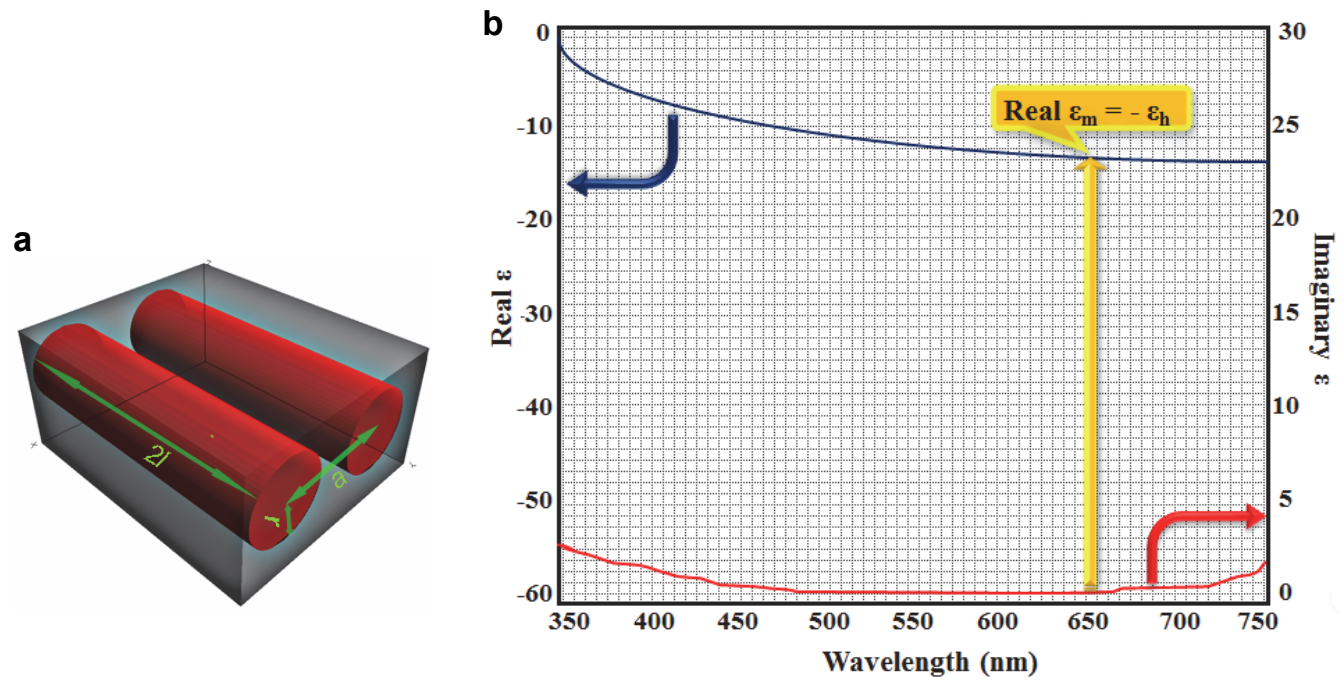

Fig. 2. Schematic of the designed LHPNA (a) and spectral outcome of electric permittivity of silver (b). Left vertical axis represents the real part of the electric permittivity, however the right vertical axis corresponds to the imaginary part of the permittivity. 
Dispersion analysis of designed LHPNA shows SHG with exact phase index matching between the primary and second harmonic wave. Further, values of effective permittivity and permeability of the proposed structure using the discrete dipole approximation for primary and second harmonic frequencies are calculated. The FDTD method is employed for a complete analysis of its propagation characteristics.

\subsection{Dispersion characteristics: negative index and SHG}

The plane wave expansion method is employed to evaluate the band diagram and equi -frequency surface (EFS) curves, shown in Figs. $3 \mathbf{a}$ and $3 \mathbf{b}$, respectively.

The following points can be noticed:

- In Fig. 3a, Juncture of a light-line with the first descending band curve of TM polarization at the normalized frequency $f(\omega a / 2 \pi c=a / \lambda)=0.23$, displays a negative unit phase index $n_{\mathrm{p}}$, signifying a possible frequency range to achieve a negative index for the designed structure with negligible losses. The firm black line in Fig. 3a articulated as light-line.

- The light-line juncture with the first eigenvalue curve specifies the utmost frequency of EFS of the designed LHPNA containing free space EFS.

- Juncture of light-line with the second ascending band at the normalized frequency $f=0.46$ parallels to the positive index at the respective second-harmonic frequency.

- From Fig. 3a it is also observed that $k_{2 \omega}-2 k_{\omega}=0$, hence a phase matching condition is achieved for SHG in the designed structure.

- The EFS of the first curve (Fig. 3b) stirring inwards with going up frequency and corresponds to LHM with $\mathbf{v}_{\mathrm{g}} \cdot \mathbf{k}_{\mathrm{f}}<0$.

Hence from Fig. 3, the absolute condition is fulfilled to accomplish all angle negative refractions. So, for all incident angles, the refracted wave vector $\mathbf{k}$ and the Poynting vector $\mathbf{S}$ are reverse in directions, which implies a negative effective index.
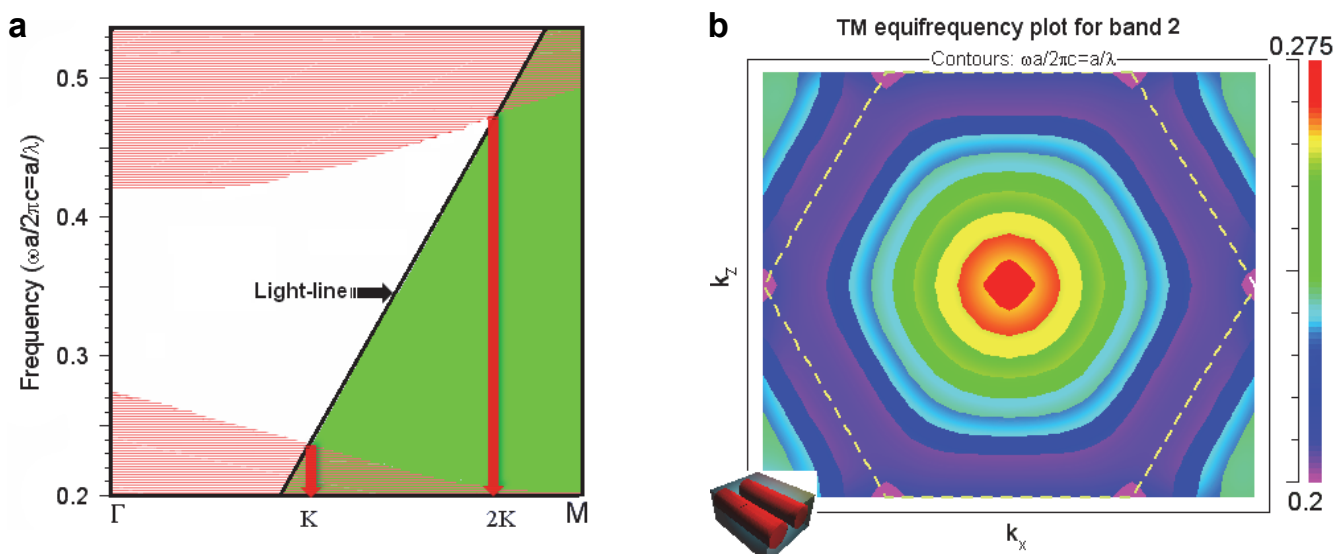

Fig. 3. Band diagram (a) and EFS corresponding descending band curve (b). Red-lines correspond to eigen values resultant in frequency curves (a). 


\subsection{Effective parameters calculation}

The calculated values of the effective permittivity and permeability are formulated in the Table for wavelengths of 650 and $325 \mathrm{~nm}$.

T a b 1 e. Permittivity and permeability values for PW $(650 \mathrm{~nm})$ and SHW wavelength $(325 \mathrm{~nm})$.

\begin{tabular}{lcc}
\hline Wavelength [nm] & Permittivity $\varepsilon$ & Permeability $\mu$ \\
\hline $650 \mathrm{~nm}$ & $-1.05349+0.0938 i$ & $-0.9487+0.78 i$ \\
$325 \mathrm{~nm}$ & $1.7641-0.012 i$ & $0.566+0.0069 i$ \\
\hline
\end{tabular}

It can be noticed that the electric permittivity and the magnetic permeability exhibit the opposite signs for both wavelengths. These values confirm that the primary wave lies in the left-handed $(\varepsilon, \mu<0)$ region, while for the second harmonic wave the right -handed $(\varepsilon, \mu>0)$ behavior appears. From the Table, it is also observed that the imaginary parts of electric permittivity $\varepsilon$ and magnetic permeability $\mu$ are ultra-low, e.g. $0.0938 i, 0.012 i$ and $0.0069 i$; and signify the minutest losses. Ignoring the imaginary part, the value of the following expression

$$
2 \pi\left[2 f\left|n_{2 \omega}\right|-2 f\left|n_{\omega}\right|\right] \Rightarrow k_{2 \omega}-2 k_{\omega} \approx 1.45 \times 10^{-4}
$$

is almost zero. Hence, the phase matching condition between the primary and $\mathrm{SH}$ wave is satisfied for a rearward generated SH wave. It is further observed that the real values of both parameters are equivalent and lead to defunct superior moments. It implies an immensely directional emission with negative refraction and supports the highly directional characteristics of the designed LHPNA.

\subsection{Mathematical analysis: second harmonic generation in LHM}

In this section, the mathematical analysis is carried out to manifest the generation of $\mathrm{SH}$ wave in the proposed LHM structure and verifying the results obtained by dispersion engineering.

Consider an optical beam $(\lambda=650 \mathrm{~nm})$ propagating along the $z$-axis in a medium with second order nonlinearity. The incident wave of frequency $\omega$ can be described as

$$
H(\omega, z)=\frac{1}{2} h(\omega, z) \exp \left(\mathrm{i} \varphi_{\omega}\right)
$$

where, $\varphi_{\omega}$ and $h(\omega, z)$ are the phase and the field amplitude, respectively.

From the dispersion analysis (Section 2.1) and effective parameters calculation (Section 2.2), it is observed that the primary frequency is in the LHM domain and the second harmonic wave is in the RHM domain. The incoming primary wave at $\omega$ refracts negatively into the nonlinear LHM and the SH wave reflects as mimicked in Fig. 4.

As an incident wave propagates in the designed structure, a nonlinear polarization $P_{2 N}=\chi^{2} E(\omega) \cdot E(\omega)$ induced is the source for SHM. 


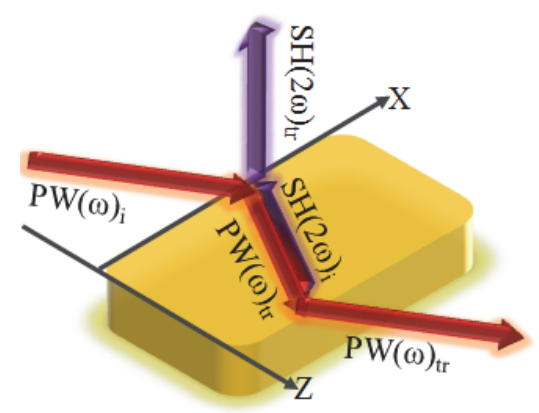

Fig. 4. Schematic of SHG in LHM. Arrows show the direction of energy flow. Indexes "i" and "tr" denote the incident and transmitted waves, respectively.

Fundamental coupled Maxwell equations explaining the gradually erratic propagation of two harmonics in LHM are given as,

$$
\begin{aligned}
& \frac{\mathrm{d} H^{2}(\omega, z)}{\mathrm{d} z^{2}}+\varepsilon(2 \omega) \mu(2 \omega) \frac{(2 \omega)^{2}}{c^{2}} H(2 \omega, z)=-\frac{(2 \omega)^{2}}{c^{2}} \chi_{\mathrm{eff}}^{2} H^{2}(\omega, z) \\
& \frac{\mathrm{d} H^{2}(\omega, z)}{\mathrm{d} z^{2}}+\varepsilon(\omega) \mu(\omega) \frac{\omega^{2}}{c^{2}} H(\omega, z)=-\frac{\omega^{2}}{c^{2}} \chi_{\mathrm{eff}}^{2} H(2 \omega, z) H^{*}(\omega, z)
\end{aligned}
$$

As the minimal signal condition, when the amplitudes of the harmonic wave alter gradually in both space and time, the basic coupled mode equation will be,

$$
\begin{aligned}
& \frac{\mathrm{d} H(2 \omega, z)}{\mathrm{d} z}=i \frac{8 \pi \varepsilon(2 \omega) \omega^{2}}{k_{2 \omega} c^{2}} g \chi_{\mathrm{eff}}^{2} H^{2}(\omega, z) \exp (-i k z) \\
& \frac{\mathrm{d} H(\omega, z)}{\mathrm{d} z}=i \frac{4 \pi \varepsilon(\omega) \omega^{2}}{2 k_{\omega} c^{2}} g \chi_{\mathrm{eff}}^{2} H^{2}(2 \omega, z) H^{*}(\omega, z) \exp (i k z)
\end{aligned}
$$

Satisfying the phase matching condition $k_{2 \omega}-2 k_{\omega}=0$, putting the negative values of permittivity $\varepsilon(\omega)$ and permeability $\mu(\omega)$, then the Manley-Rowe relation for LHM becomes,

$$
|H(\omega, z)|^{2}-|H(2 \omega, z)|^{2}=\text { const }
$$

Employing the boundary conditions, $h_{\omega}(0)=h_{\omega 0}$ and $h_{2 \omega}(L)=0$, after arduous calculations, the solution of the equation can be described as,

$$
h(2 \omega, z)=\zeta \tan [\zeta \rho(L-z)]
$$

This expression represents the field distribution of the second harmonic wave in LHM. Here 


$$
\zeta=\sqrt{h^{2}(\omega, z)-h^{2}(2 \omega, z)}
$$

with

$$
\begin{aligned}
& h^{2}(2 \omega, z)=0 \\
& \rho=\frac{8 \pi \varepsilon(2 \omega) \omega^{2}}{k_{2 \omega} c^{2}} \chi_{\mathrm{eff}}^{(2)}
\end{aligned}
$$

The field distribution of the primary wave in LHM is as follows,

$$
h(\omega, z)=\zeta \sec [\zeta \rho(L-z)]
$$

The values of effective parameters put in Eqs. (7) and (8) provide the field intensity distribution plot in LHM as shown in Figs. 5a and 5b. The effective value of the second order nonlinearity $\chi_{\text {eff }}^{(2)}$ for designed LHPNA is chosen to be $170 \mathrm{pm} / \mathrm{V}$. From Fig. 5a it can be noted that intensity goes down for both waves along the $z$-axis and tends towards the minimum value when $z=L$. Disparity is also observed among the normalized

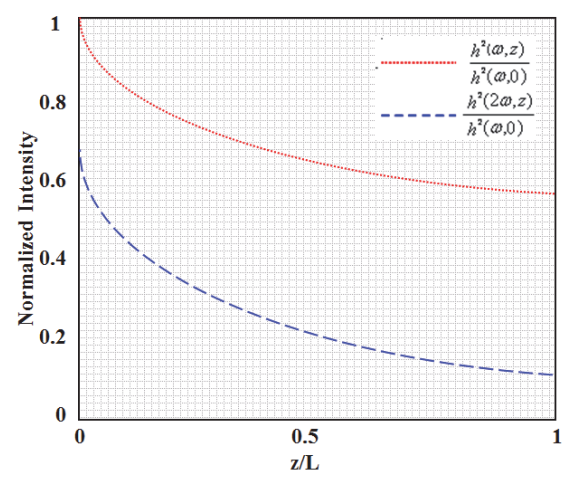

a

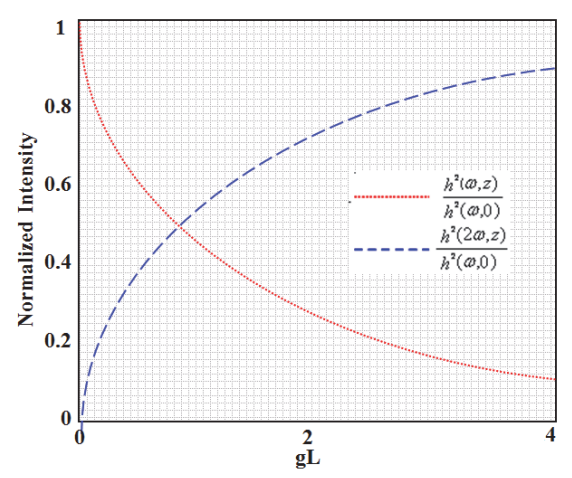

b

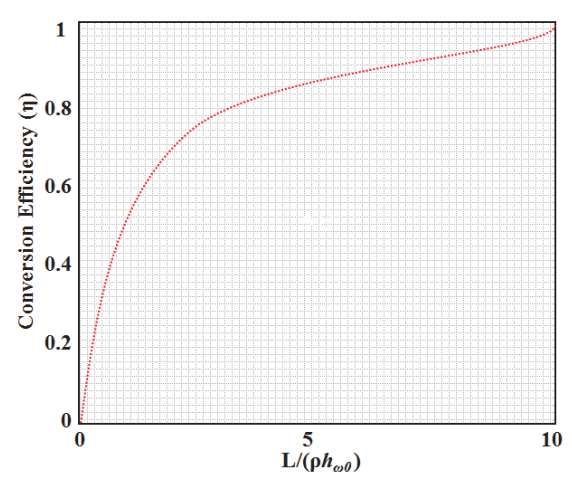

C

Fig. 5. Dependence of the field intensity plot of the primary and second harmonic wave. Inside the slab width of designed LHPNA $g L=1$ (a); primary wave at $z=L$ and SH at $z=0$ (b); conversion efficiency with respect to normalized thickness of designed LHPNA (c). 
field intensity distributions of the primary and the $\mathrm{SH}$ wave, and varies with static LHPNA width $(g L=1)$ and persists the same inside the entire LHPNA. It is observed that disparity between the primary wave and the second harmonic wave decreases with increasing $g L$. This confirms that conservation efficiency also increases as shown in Fig. $5 \mathbf{c}$. Figure $5 \mathbf{b}$ reveals the dependence of the primary wave and the second harmonic wave with variation of slab width. Here the intensity of SH wave (UV light) goes up with the increasing slab thickness and vice versa for the PW (red light). It is observed that conversion efficiency goes up with increasing primary wave intensity. Furthermore, Fig. 5c indicates conversion efficiency with variation of normalized slab width. Here, conversion efficiency increases with increasing slab width. It is revealed that conversion efficiency tends to the maximum value as slab width $L$ goes to infinite.

\section{Characterization}

In order to further characterize the designed LHPNA, the transmission spectrum of the device is calculated using the 3D FDTD method. The transmission spectrum is depicted in Fig. 6, whereas the corresponding field map is illustrated in the inset of Fig. 6 . Through the field map, we can visualize the negative refraction and the generation of the SH wave in the designed structure, which is confirmed by the respective transmitted primary wave (solid red curve) and reflected SH wave (dashed blue curve) with transmitted SH wave (solid blue curve) spectrums in the adjoined Fig. 6a. The incident red light $(\lambda=650 \mathrm{~nm})$ exhibits $>60 \%$ transmission in a left-handed regime, whereas the generated SH wave corresponding to the ultraviolet light $(\lambda=325 \mathrm{~nm})$ shows $>70 \%$ of reflection and up to 255 transmissions from the structure. Note that, the generated
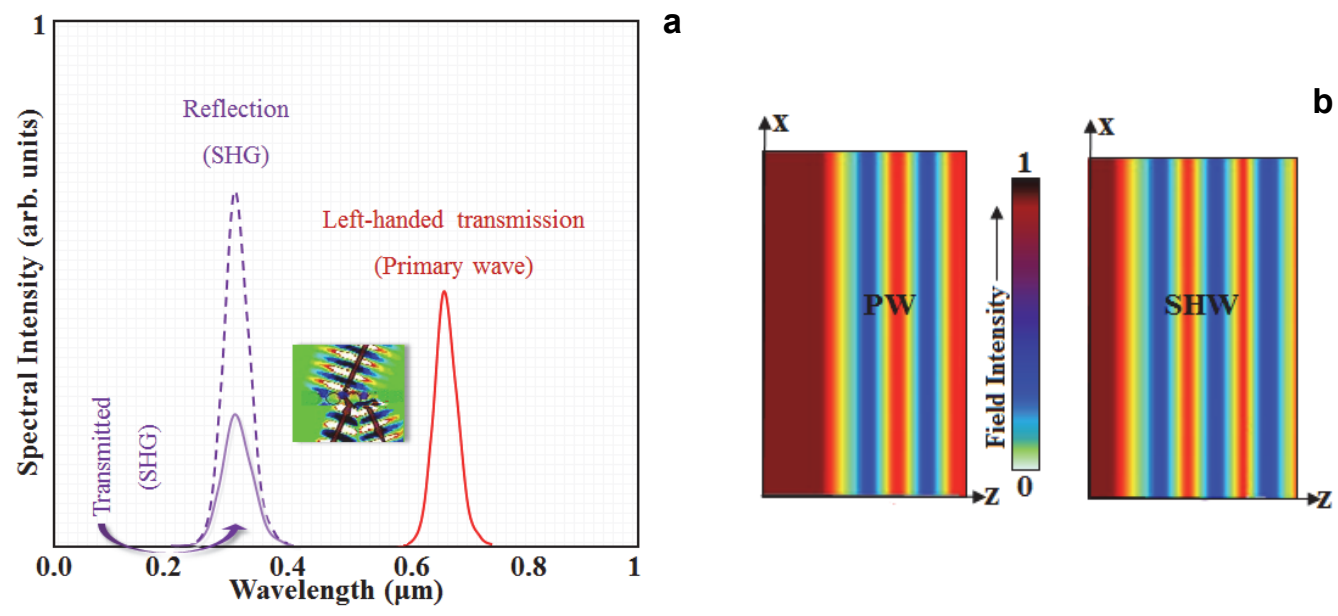

Fig. 6. Transmission and reflection response with wavelength, for the designed LHPNA, where left-handed transmission for red light and reflection as well as transmission for emitted UV light are shown with the field atlas in inset (a). Field distribution for the incident primary wave (PW) or red light and reflected second harmonic wave (SHW) or UV light inside the designed LHPNA (b). 
and transmitted waves possess TM polarization and the structural parameters are given as the thickness of the LHPNA slab $L=65 \mathrm{~nm}, r=31 \mathrm{~nm}, a=150 \mathrm{~nm}$. Results obtained in transmission spectra are in virtuous harmony with the result obtained in the numerical analysis. Figure $6 \mathbf{b}$ exhibits the field model inside the designed LHPNA for a static slab width $(L=65 \mathrm{~nm})$. It is perceived that the field intensity for red light (PW) is decreasing width of designed LHPNA (along $z$-axis). However, reflected field intensity of generated UV light (SHW) is increasing along the axis. This reconfirms the results obtained by the numerical analysis and transmission spectra.

\subsection{Flat lens application}

Further, Fig. 7 demonstrated the flat lens application of the designed structure. This issue in LHM has been earlier discussed by the researchers, for the microwave and the infrared region. In present paper, the imaging effect for the red light region with a centered wavelength at $\lambda=650 \mathrm{~nm}$ from the simplest design of LHM with no aberration is at-
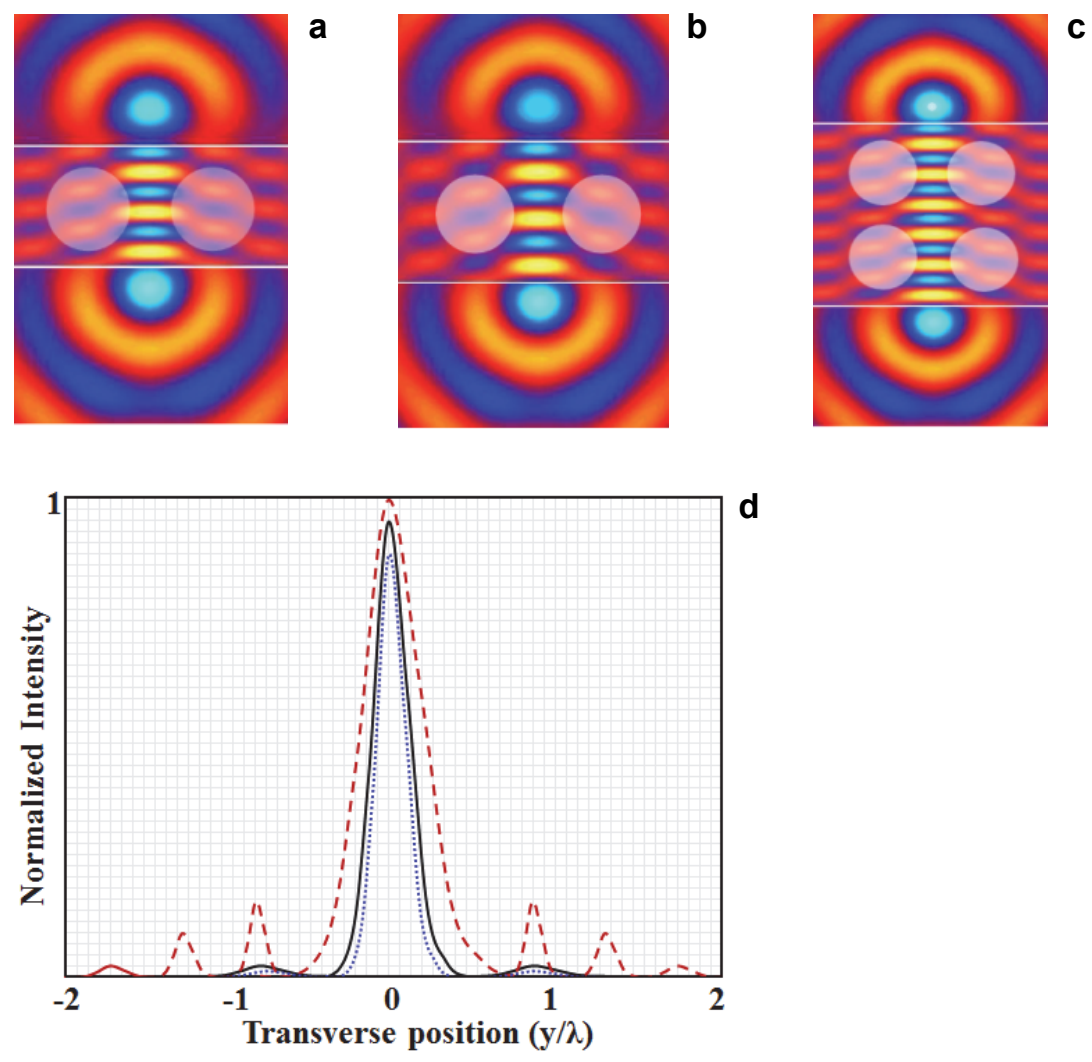

Fig. 7. Field atlas of imaging from the designed structure $(L=65 \mathrm{~nm})(\mathbf{a})$, increasing host GaAs thickness $(L=75 \mathrm{~nm})(\mathbf{b})$, and increasing layer of nanorods (c). Normalized intensity distribution of the image plane along the transverse coordinate for designed lens with a solid curve for $L=65 \mathrm{~nm}$, dashed curve for the increased layer of a nanorod system and dotted curve for increasing host material thickness (d). 
tained. In this section, the analysis of the focusing property of the designed LHM with an effective index $n_{\text {eff }} \approx-1$ (from Sections 2.1 and 2.2) is carried out for structure modification. Figure 7 demonstrated the imaging effect for the designed structure with structural modification. To attain the imaging, we consider a TM polarized point source, centered at one side $(z=-u)$ of the lens, while the two surfaces of the slab are placed at $z=0$ and $z=L$ (here $L=65 \mathrm{~nm}$ ). An image is formed on the other side of the lens as shown in Fig. 7a. In Fig. 7b, thickness of host material GaAs increases, which results in reduced intensity of the image spot. In Fig. 7c, the imaging effect with an increased layer of nanorods is observed. It is revealed that intensity of the image increases with an increased width.

The image inside the designed structure is not visible due to the small thickness of a slab. Normalized intensity distribution of the image plane along the transverse coordinate for designed lens is also shown by Fig. $7 \mathbf{d}$. A focal point containing a crosswise spot size less than one is generated by the designed flat lens. This indorsed the negligible spherical aberrations. Furthermore, the dependence of the focusing properties of the designed LHPNA on the increased slab thickness $(L=75 \mathrm{~nm})$ and layer of nanorod inclusion is also shown in Figs. $7 \mathbf{b}$ and $7 \mathbf{d}$. By comparing the simulation results of the designed structure with different slab thickness, from Figs. $7 \mathbf{a}, 7 \mathbf{b}$ and $7 \mathbf{c}, 7 \mathbf{d}$, it is observed that the formed image dwindles with increasing thickness of the designed LHPNA.

\subsection{Optical plasmonic nanoantenna}

Finally, further characterization of the designed LHPNA to strengthen its authentication is performed. In Section 2.2, it is also observed that the real values of both the electric permittivity and the magnetic permeability are equivalent and lead to defunct superior moments, which implies an immensely directional emission with negative refraction. In the present section, we have evaluated the far-field spectrum and LHTE of the designed structure to reinforce the above mentioned conclusion.

\subsubsection{Far-field spectrum and left-handed transmission efficiency}

Figures $8 \mathbf{a}$ and $8 \mathbf{b}$ are the far-field spectrums obtained by employing the 3D FDTD method. Angular distribution of intensity at the output of the designed LHM is shown in Figs. $8 \mathbf{a}$ and $8 \mathbf{b}$. It is observed that the transmission peak (pink loop) is obtained in the deep negative side (same side to the normal of second interface), which confirms highly directional left-handed transmission from the designed LHPNA. A green loop in Fig. 8a, shows the transmission peak in right-handed direction, also known as right -handed transmission. 3D far field intensity profile again reconfirmed highly directional left-handed transmission.

In LHM, even though transmission is dominated by a negative refraction (NR), a component of positive refraction (PR) is always associated with it, confirming the presence of some radiation towards the positive side of the left-handed structure. Incident angle dependence of left-handed transmission (NR) and right-handed transmission (PR) is shown in Fig. 8c, where NR is achieved for a broad range of incident angles with ultra -low right-handed transmission. In order to confirm the validity of the proposed struc- 

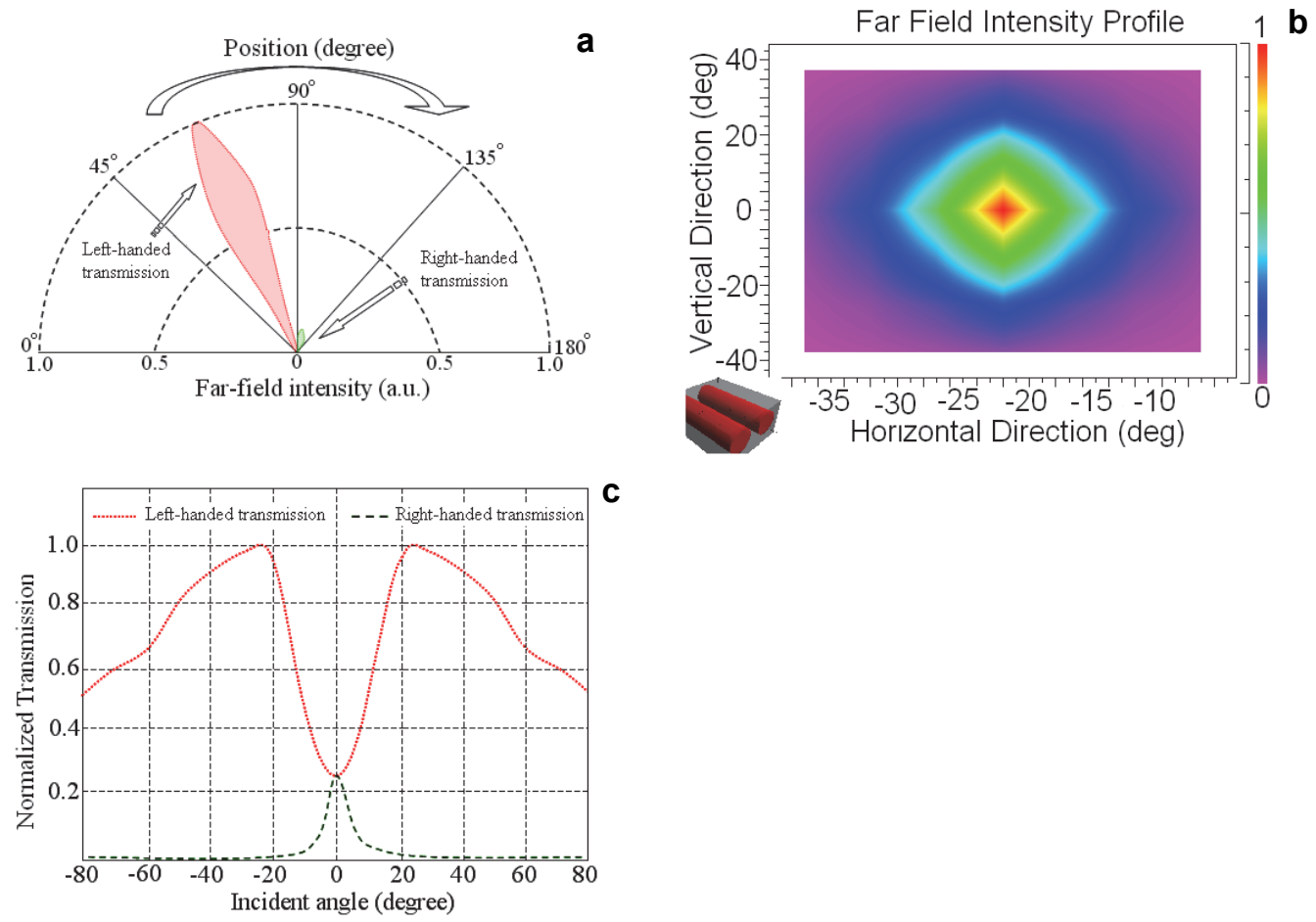

Fig. 8. Far-field intensity pattern $v s$. horizontal position (deg) of TM mode at $\lambda=650 \mathrm{~nm}$, which shows negative refraction for the designed LHM $(\mathbf{a}, \mathbf{b})$. Angular response of left-handed and right-handed transmission in the designed LHM (c).

ture as a LHM, the left-handed transmission efficiency (LHTE) is calculated according to the following equation:

$$
\text { LHTE }=\frac{T_{\mathrm{NR}}-T_{\mathrm{PR}}}{T_{\mathrm{NR}}+T_{\mathrm{PR}}} \times 100 \%
$$

where $T_{\mathrm{NR}}$ denotes transmission in negative direction, and $T_{\mathrm{PR}}-$ transmission in positive direction. LHTE in excess of $98 \%$ is calculated. Immense LHTE authenticates the highly directional nanoantenna characteristics of the designed structure.

\section{Conclusion}

A tailored index matched LHM is designed for demonstration of ultraviolet emission through SHG. By investigation of the dispersion properties and effective parameters (negative values of permeability and permittivity), it is demonstrated that the designed structure is left-handed for the primary wave and right-handed for the second harmonic wave. The outcomes of a dispersion analysis are mightily supported by the effective parameters investigation. Further, the obtained results by numerical calculation for 
SHG are in good agreement with the FDTD analysis. Moreover, flat lens application with no aberration is also displayed for a red light region. Its nanoantenna application is firmly supported by the far field analysis and left-handed transmission efficiency, which is over and above $98 \%$. The study of the angular response of left-handed and right-handed transmission for the designed LHM validates its authentication for a large range of incident angles. Eventually, a multifunction LHM is designed unveiling tremendous potential to amass and abridge future structures in one architecture.

Acknowledgements - The authors gratefully acknowledge, Prof. R.K. Sinha for his immense suggestions and support of TIFAC Centre of Relevance and Excellence in Fiber Optics and Optical Communication at Delhi Technological University (Formerly Delhi College of Engineering), Delhi through Mission REACH program of Technology Vision-2020, Government of India.

\section{References}

[1] TAYA S.A., P-polarized surface waves in a slab waveguide with left-handed material for sensing applications, Journal of Magnetism and Magnetic Materials 377, 2015, pp. 281-285, DOI: $10.1016 /$ j.jmmm.2014.10.126.

[2] Yao J., Liu Z., Liu Y., Wang Y., Sun C., Bartal G., Stacy A.M., Zhang X., Optical negative refraction in bulk materials of nanowires, Science 321(5891), 2008, p. 930, DOI: 10.1126/science.1157566.

[3] Taya S.A., El-Agez T.M., Kullab H.M., Abadla M.M., Shabat M.M., Theoretical study of slab waveguide optical sensor with left-handed material as a core layer, Optica Applicata 42(1), 2012, pp. 193-205, DOI: 10.5277/oa120118.

[4] Yuan H.-K., Chettiar U.K., Cai W., Kildishev A.V., Boltasseva A., Drachev V.P., Shalaev V.M., A negative permeability material at red light, Optics Express 15(3), 2007, pp. 1076-1083, DOI: $\underline{10.1364 / O E .15 .001076 .}$.

[5] Zhang S., Fan W., Panoiu P.C., Malloy K.J., Osgood R.M., Brueck S.R.J., Demonstration of nearinfrared negative-index metamaterials, Physical Review Letters 95(13), 2005, article ID 137404, DOI: 10.1103/PhysRevLett.95.137404.

[6] Taya S.A., Mahdi S.S., Alkanoo A.A., QAdoura I.M., Slab waveguide with conducting interfaces as an efficient optical sensor: TE case, Journal of Modern Optics 64(8), 2017, pp. 836-843, DOI: 10.1080/09500340.2016.1262072.

[7] Taya S.A., Jarada A.A., Kullab H.M., Slab waveguide sensor utilizing left-handed material core and substrate layers, Optik 127(19), 2016, pp. 7732-7739, DOI: 10.1016/j.ijleo.2016.05.095.

[8] Algredo-Badillo U., Halevi P., Negative refraction and focusing in magnetically coupled L-C loaded transmission lines, Journal of Applied Physics 102(8), 2007, article ID 086104, DOI: $\underline{10.1063 /}$ 1.2794558.

[9] Prayakarao S., Robbins S., Kinsey N., Boltasseva A., Shalaev V.M., Wiesner U.B., Bonner C.E., Hussain R., Noginova N., Noginov M.A., Gyroidal titanium nitride as nonmetallic metamaterial, Optical Materials Express 5(6), 2015, pp. 1316-1322, DOI: 10.1364/OME.5.001316.

[10] Govyadinov A.A., Podolskiy V.A., Active metamaterials: sign of refractive index and gain-assisted dispersion management, Applied Physics Letters 91(19), 2007, article ID 191103, DOI: $10.1063 /$ 1.2800309.

[11] Behera G., Ramakrishna S.A., Tri-layered composite plasmonic structure with a nanohole array for multiband enhanced absorption at visible to NIR frequencies: plasmonic and metamaterial resonances, Journal of Physics D: Applied Physics 49(7), 2016, article ID 075103, DOI: $10.1088 /$ $\underline{0022-3727 / 49 / 7 / 075103 .}$. 
[12] RajPUT M., SiNHA R.K., All-angle negative refraction for visible light from left-handed metallo-dielectric photonic crystal: theoretical and numerical demonstration with nanophotonic device application, Applied Physics B 98(1), 2010, pp. 99-106, DOI: 10.1007/s00340-009-3685-7.

[13] Nehmetallah G., Aylo R., Powers P., Sarangan A., Gao J., Li H., Achari A., Banerjee P.P., Co-sputtered $\mathrm{SiC}+\mathrm{Ag}$ nanomixtures as visible wavelength negative index metamaterials, Optics Express 20(7), 2012, pp. 7095-7100, DOI: 10.1364/OE.20.007095.

[14] Zhou J., Koschny Th., Kafesaki M., Economou E.N., Pendry J.B., Soukoulis C.M., Saturation of magnetic response of split-ring resonators at optical frequencies, Physical Review Letters 95(22), 2005, article ID 223902, DOI: 10.1103/PhysRevLett.95.223902.

[15] TAYA S.A., Theoretical investigation of slab waveguide sensor using anisotropic metamaterial, Optica Applicata 45(3), 2015, pp. 405-417, DOI: 10.5277/oa150312.

[16] Abadla M.M., TAYa S.A., Theoretical investigation of guided modes in planar waveguides having chiral negative index metamaterial core layer, Optik 131, 2017, pp. 562-573, DOI: 10.1016/j.ijleo. 2016.11.184.

[17] Taya S.A., Dispersion properties of lossy, dispersive, and anisotropic left-handed material slab waveguide, Optik 126(14), 2015, pp. 1319-1323, DOI: 10.1016/j.ijleo.2015.04.013.

[18] Lapine M., Gorkunov M., Ringhofer K.H., Nonlinearity of a metamaterial arising from diode insertions into resonant conductive elements, Physical Review E 67(6), 2003, article ID 065601(R), DOI: 10.1103/PhysRevE.67.065601.

[19] Shadrivov I.V., Zharov A.A., Kivshar Y.S., Second-harmonic generation in nonlinear left-handed metamaterials, Journal of the Optical Society of America B 23(3), 2006, pp. 529-534, DOI: 10.1364/ JOSAB.23.000529.

[20] Popov A.K., Slabko V.V., Shalaev V.M., Second harmonic generation in left-handed materials, Laser Physics Letters 3(6), 2006, pp. 293-297, DOI: 10.1002/lapl.200610008.

[21] D’Aguanno G., Mattiucci N., Bloemer M.J., Scalora M., Large enhancement of second harmonic generation near the zero- $\bar{n}$ gap of a negative-index Bragg grating, Physical Review E 73(3), 2006, article ID 036603, DOI: 10.1103/PhysRevE.73.036603.

[22] Rajput M., Sinha R.K., Rawal S., Varshney S.K., UV emission from left-handed material through second harmonic generation: optical nanoantenna and imaging application, Micro and Nano Letters 6(8), 2011, pp. 575-578, DOI: 10.1049/mnl.2011.0171.

[23] Banerjee P.P., Nehmetallah G., Linear and nonlinear propagation in negative index materials, Journal of the Optical Society of America B 23(11), 2006, pp. 2348-2355, DOI: 10.1364/JOSAB.23. 002348.

[24] Popov A.K., Salaev V.M., Negative-index metamaterials: second-harmonic generation, Maney-Rowe relations and parametric amplification, Applied Physics B 84(1-2), 2006, pp. 131-137, DOI: 10.1007 / s00340-006-2167-4.

[25] Klein M.W., EnKrich C., Wegener M., Linden S., Second-harmonic generation from magnetic metamaterials, Science 313(5786), 2006, pp. 502-504, DOI: 10.1126/science.1129198.

[26] Gorkunov M.V., Shradivov I.V., Kivshar Y.S., Enhanced parametric processes in binary metamaterials, Applied Physics Letters 88(7), 2006, article ID 071912, DOI: 10.1063/1.2168755.

[27] Han J., LAKHTAKIA A., QIU C.-W., Terahertz metamaterials with semiconductor split-ring resonators for magnetostatic tunability, Optics Express 16(19), 2008, pp. 14390-14396, DOI: 10.1364/OE.16. 014390.

[28] Sarau G., Lahiri B., Banzer P., Gupta P., Bhattacharya A., Vollmer F., Christiansen S., Enhanced Raman scattering of graphene using arrays of split ring resonators, Advanced Optical Materials 1(2), 2013, pp. 151-157, DOI: 10.1002/adom.201200053.

[29] Kudyshev Zh., Gabitov I., Maimistov A., Effect of phase mismatch on second-harmonic generation in negative-index materials, Physical Review A 87(6), 2013, article ID 063840, DOI: $10.1103 /$ PhysRevA.87.063840. 
[30] Chandrasekar R., Emani Naresh K., Lagutchev A., Shalaev V.M., Ciracì C., Smith D.R., KILDISHEV A.V., Second harmonic generation with plasmonic metasurfaces: direct comparison of electric and magnetic resonances, Optical Materials Express 5(11), 2015, pp. 2682-2691, DOI: 10.1364/OME.5.002682.

[31] Guddala S., Narayana Rao D., Ramakrishna S.A., Resonant enhancement of Raman scattering in metamaterials with hybrid electromagnetic and plasmonic resonances, Journal of Optics 18(6), 2016, article ID 065104, DOI: 10.1088/2040-8978/18/6/065104.

[32] Maas R.,Verhagen E., Parsons J., Polman A., Negative refractive index and higher-order harmonics in layered metallodielectric optical metamaterials, ACS Photonics 1(8), 2014, pp. 670-676, DOI: 10.1021/ph5000874.

Received September 10, 2018 in revised form October 23, 2018 